\title{
Sivers Asymmetry and Generalized Parton Distributions in Impact Parameter Space
}

\author{
Matthias Burkardt \\ Department of Physics, New Mexico State University, \\ Las Cruces, NM 88003-0001, U.S.A. \\ Dae Sung Hwang \\ Department of Physics, Sejong University, \\ Seoul 143-747, Korea
}

September 3, 2018

\begin{abstract}
Recently, it has been pointed out that there exists a connection between the generalized parton distribution $E(x, 0, t)$ and the Sivers asymmetry. For transversely polarized nucleon targets, generalized parton distributions are asymmetric in impact parameter space. This impact parameter space asymmetry, together with the final state interaction of the active quark, gives rise to the Sivers asymmetry in momentum space. We demonstrate this phenomenon explicitly in the scalar diquark model. This result also illustrates the physics that underlies the correlation between the anomalous magnetic moment and the Sivers asymmetry for a given quark flavor.
\end{abstract}




\section{Introduction}

Recently, one gluon exchange in the final state interactions (FSI) has been suggested [1] as a mechanism for generating a transverse single-spin asymmetry (SSA) in semiinclusive deep inelastic scattering processes. This FSI can be effectively taken into account by introducing an appropriate Wilson line phase factor in the definition of the distribution functions of quarks in the nucleon $[2,3,4,5]$. The physical interpretation of this Wilson line phase factor is that it describes the phase factor of the propagator for the active quark as it leaves the target. Therefore, the Wilson line extends from the position of the quark along a light-like future-oriented direction. This phase factor is not invariant under time-reversal, which is the reason why the Sivers asymmetry can be nonzero when the phase factor is included.

This mechanism has a nice physical interpretation in transverse position space, where one can show that the Sivers asymmetry arises from a left-right (relative to the nucleon spin) asymmetry of the quark distribution in impact parameter space. The Wilson line phase factor describes the effect of the transverse component of the force that is acting on the active quark after it is knocked out of the target. This force is on average directed towards the center of the nucleon. This chromodynamic lensing effect thus translates the T-even transverse position space asymmetry into a T-odd transverse momentum space asymmetry of the leading quark [6, 7], which is the Sivers asymmetry.

In Ref. [1] a simple scalar diquark model was used to demonstrate explicitly that the FSI can indeed give rise to a leading-twist transverse SSA, which emerged from interference between spin dependent amplitudes with different nucleon spin states. In Refs. [1, 8] it was observed that the same overlap integrals between light-cone wavefunctions that describe the anomalous magnetic moment contribution from a given quark flavor also appear in the Sivers distribution for that quark flavor (with additional pieces in the integrand). Since these integrals are the overlaps between light-cone wavefunctions whose orbital angular momenta differ by $\Delta L^{z}= \pm 1$, the 
orbital angular momentum of the quark inside the proton is essential for the existence of the Sivers asymmetry. In Refs. [6, 7], the transverse distortion of impact parameter dependent parton distributions for transversely polarized targets in the infinite momentum frame, was used to develop a physical explanation for the sign of the Sivers asymmetry. In this paper, we diagonalize the relevant amplitudes in Ref. [1] by transforming to an impact parameter space basis [7]*. This should not only yield a clear intuitive physical interpretation for the SSA derived in Ref. [1], but at the same time provide an explicit example that illustrates a proposed connection between generalized parton distributions and SSAs [7].

\section{GPDs in impact parameter space (scalar diquark model)}

Light-cone wavefunctions provide a very convenient representation of generalized parton distributions (GPDs) in terms of overlap integrals [13, 14]. For example, in a scalar diquark model in which a nucleon is modeled as a bound state of a quark and a scalar diquark, one finds in the case $\xi=0$ (purely transverse momentum transfer $\left.\Delta=p-p^{\prime}\right)$

$$
\begin{aligned}
& H\left(x, 0,-\Delta_{\perp}^{2}\right) \\
& \quad=\frac{1}{4 \pi} \int \frac{d^{2} \mathbf{k}_{\perp}}{(2 \pi)^{2}}\left[\widetilde{\psi}_{+\frac{1}{2}}^{\uparrow}\left(x, \mathbf{k}_{\perp}^{\prime}\right) \widetilde{\psi}_{+\frac{1}{2}}^{\uparrow}\left(x, \mathbf{k}_{\perp}\right)+\widetilde{\psi}_{-\frac{1}{2}}^{\uparrow}\left(x, \mathbf{k}_{\perp}^{\prime}\right) \widetilde{\psi}_{-\frac{1}{2}}^{\uparrow}\left(x, \mathbf{k}_{\perp}\right)\right] \\
& \frac{\left(\Delta^{x}-i \Delta^{y}\right)}{2 M} E\left(x, 0,-\Delta_{\perp}^{2}\right) \\
& \quad=\frac{1}{4 \pi} \int \frac{d^{2} \mathbf{k}_{\perp}}{(2 \pi)^{2}}\left[\widetilde{\psi}_{+\frac{1}{2}}^{\uparrow *}\left(x, \mathbf{k}_{\perp}^{\prime}\right) \widetilde{\psi}_{+\frac{1}{2}}^{\downarrow}\left(x, \mathbf{k}_{\perp}\right)+\widetilde{\psi}_{-\frac{1}{2}}^{\uparrow}\left(x, \mathbf{k}_{\perp}^{\prime}\right) \widetilde{\psi}_{-\frac{1}{2}}^{\downarrow}\left(x, \mathbf{k}_{\perp}\right)\right]
\end{aligned}
$$

where

$$
\mathbf{k}_{\perp}^{\prime}=\mathbf{k}_{\perp}-(1-x) \boldsymbol{\Delta}_{\perp}
$$

*For a general discussion of parton distributions in impact parameter space and their connection to generalized parton distributions the reader is refered to Refs. [9, 10, 11, 12]. 
For higher Fock components, similar convolution formulas exist [13, 14].

A well known feature of Fourier transforms is that they diagonalize convolution integrals. For this purpose we switch to the transverse position space representation of the light-cone wavefunction

$$
\psi_{s}^{\lambda}\left(x, \mathbf{c}_{\perp}\right) \equiv \int \frac{d^{2} \mathbf{k}_{\perp}}{(2 \pi)^{2}} e^{i \mathbf{k}_{\perp} \mathbf{c}_{\perp}} \tilde{\psi}_{s}^{\lambda}\left(x, \mathbf{k}_{\perp}\right)
$$

Note that the transverse momentum $\mathbf{k}_{\perp}$ in the two-particle Fock component is Fourier conjugate to the distance $\mathbf{c}_{\perp} \equiv \mathbf{r}_{\perp 1}-\mathbf{r}_{\perp 2}$ between the active quark and the spectator. However, since GPDs have a particularly simple form in impact parameter representation, we change variables from $\mathbf{c}_{\perp}$ to the impact parameter $\mathbf{b}_{\perp}$ (the distance between the active quark and the center of longitudinal momentum), which are related by

$$
\mathbf{b}_{\perp}=(1-x) \mathbf{c}_{\perp}
$$

yielding

$$
\begin{aligned}
\mathcal{H}\left(x, \mathbf{b}_{\perp}\right) & \equiv \int \frac{d^{2} \boldsymbol{\Delta}_{\perp}}{(2 \pi)^{2}} e^{i \boldsymbol{\Delta}_{\perp} \cdot \mathbf{b}_{\perp}} H\left(x, 0,-\boldsymbol{\Delta}_{\perp}^{2}\right) \\
& =\frac{1}{4 \pi}\left[\psi_{+\frac{1}{2}}^{\uparrow *}\left(x, \mathbf{c}_{\perp}\right) \psi_{+\frac{1}{2}}^{\uparrow}\left(x, \mathbf{c}_{\perp}\right)+\psi_{-\frac{1}{2}}^{\uparrow *}\left(x, \mathbf{c}_{\perp}\right) \psi_{-\frac{1}{2}}^{\uparrow}\left(x, \mathbf{c}_{\perp}\right)\right] \frac{1}{(1-x)^{2}} .
\end{aligned}
$$

Likewise one finds

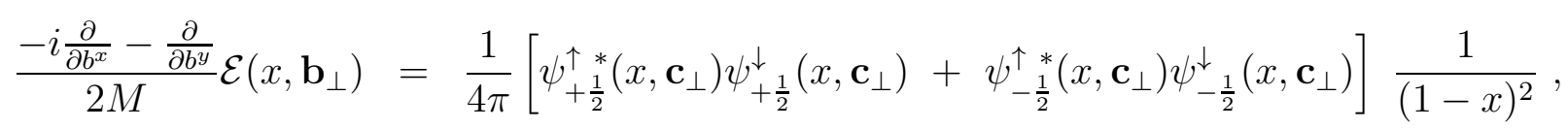

where

$$
\mathcal{E}\left(x, \mathbf{b}_{\perp}\right) \equiv \int \frac{d^{2} \boldsymbol{\Delta}_{\perp}}{(2 \pi)^{2}} e^{i \boldsymbol{\Delta}_{\perp} \cdot \mathbf{b}_{\perp}} E\left(x, 0,-\boldsymbol{\Delta}_{\perp}^{2}\right) .
$$

The physical significance of $\mathcal{E}\left(x, \mathbf{b}_{\perp}\right)$, i.e., the spin-flip distribution in impact parameter space becomes clear when we consider a state that is polarized in the $+\hat{y}$ direction (in the infinite momentum frame)

$$
\left|P^{+}, \mathbf{R}_{\perp}=\mathbf{0}_{\perp},+\hat{y}\right\rangle \equiv \frac{1}{\sqrt{2}}\left(\left|P^{+}, \mathbf{R}_{\perp}=\mathbf{0}_{\perp}, \uparrow\right\rangle+i\left|P^{+}, \mathbf{R}_{\perp}=\mathbf{0}_{\perp}, \downarrow\right\rangle\right) .
$$


For this state, the unpolarized quark distribution in impact parameter space reads

$$
\begin{aligned}
q_{\hat{y}}\left(x, \mathbf{b}_{\perp}\right) & \equiv\left\langle P^{+}, \mathbf{R}_{\perp}=\mathbf{0}_{\perp},+\hat{y}\left|\hat{O}_{q}\left(x, \mathbf{b}_{\perp}\right)\right| P^{+}, \mathbf{R}_{\perp}=\mathbf{0}_{\perp},+\hat{y}\right\rangle \\
& =\int \frac{d^{2} \boldsymbol{\Delta}_{\perp}}{(2 \pi)^{2}} e^{i \boldsymbol{\Delta}_{\perp} \cdot \mathbf{b}_{\perp}}\left[H\left(x, 0,-\boldsymbol{\Delta}_{\perp}^{2}\right)+i \frac{\Delta^{x}}{2 M} E\left(x, 0,-\boldsymbol{\Delta}_{\perp}^{2}\right)\right] \\
& =\mathcal{H}\left(x, \mathbf{b}_{\perp}\right)+\frac{1}{2 M} \frac{\partial}{\partial b^{x}} \mathcal{E}\left(x, \mathbf{b}_{\perp}\right) .
\end{aligned}
$$

We will write in Section 4 on the interesting (and important) property that the density in impact parameter space $q_{\hat{y}}\left(x, \mathbf{b}_{\perp}\right)$ has the asymmetry along the $\hat{x}$-direction in impact parameter space when the proton spin is polarized along the $\hat{y}$-direction as we see in (10), whereas there does not exist such an asymmetry for the density in momentum space.

\section{Transverse distortion of the wavefunction}

In Ref. [12] it was shown that if the spin-flip generalized parton distribution $E_{q}\left(x, 0,-\Delta_{\perp}^{2}\right)$ is nonzero, then the parton distribution of quarks with flavor $q$ is distorted in the transverse plane when the target has a transverse polarization. For a nucleon with spin pointing in the positive $\hat{y}$-direction, one finds $[12]$

$$
q_{\hat{y}}\left(x, \mathbf{b}_{\perp}\right)=\mathcal{H}\left(x, \mathbf{b}_{\perp}\right)+\frac{1}{2 M} \frac{\partial}{\partial b^{x}} \mathcal{E}\left(x, \mathbf{b}_{\perp}\right),
$$

where $\mathcal{H}$ and $\mathcal{E}$ are Fourier transforms of generalized parton distributions

$$
\begin{aligned}
\mathcal{H}_{q}\left(x, \mathbf{b}_{\perp}\right) & \equiv \int \frac{d^{2} \boldsymbol{\Delta}_{\perp}}{(2 \pi)^{2}} e^{i \boldsymbol{\Delta}_{\perp} \cdot \mathbf{b}_{\perp}} H_{q}\left(x, 0,-\boldsymbol{\Delta}_{\perp}^{2}\right) \\
\mathcal{E}_{q}\left(x, \mathbf{b}_{\perp}\right) & \equiv \int \frac{d^{2} \boldsymbol{\Delta}_{\perp}}{(2 \pi)^{2}} e^{i \boldsymbol{\Delta}_{\perp} \cdot \mathbf{b}_{\perp}} E_{q}\left(x, 0,-\boldsymbol{\Delta}_{\perp}^{2}\right) .
\end{aligned}
$$

One of the things that are known about $\mathcal{E}_{q}$ is that its integral should give the contribution from flavor $q$ to the anomalous magnetic moment ${ }^{\dagger}$

$$
\int d x \int d^{2} \mathbf{b}_{\perp} \mathcal{E}_{q}\left(x, \mathbf{b}_{\perp}\right)=\kappa_{q}
$$

\footnotetext{
${ }^{\dagger}$ The measured magnetic moment of the proton is obtained as a superposition from all quark flavors $\kappa_{p}=\sum_{q} e_{q} \kappa_{q}=1.79$.
} 
Integrating $E\left(x, 0,-\boldsymbol{\Delta}_{\perp}^{2}\right)$ over $x$ yields the Pauli form factor $F_{2}\left(-\boldsymbol{\Delta}_{\perp}^{2}\right)$. Therefore, unless the $x$ dependence introduces a fluctuating sign into $E\left(x, 0,-\boldsymbol{\Delta}_{\perp}^{2}\right)$, the sign of $F_{2}$ thus determines the sign of $E\left(x, 0,-\Delta_{\perp}^{2}\right)$. Hence one expects that $E\left(x, 0,-\Delta_{\perp}^{2}\right)$ is a smooth function of $\boldsymbol{\Delta}_{\perp}$ with a maximum (or minimum if $\kappa_{q}<0$ ) at the origin. From experience with Fourier transforms it is thus clear that $\mathcal{E}\left(x, \mathbf{b}_{\perp}\right)$ also has a maximum (minimum) at the origin and is otherwise a smooth function that will have the same sign as $\kappa_{q}$ for all (or most) values of $\mathbf{b}_{\perp}$.

The $b^{x}$-derivative of a smooth positive function $\mathcal{E}\left(x, \mathbf{b}_{\perp}\right)$ with maximum at the origin (we consider here the case $\kappa_{q}>0$ ) is positive for negative $b^{x}$ and negative for positive $b^{x}$. For valence quarks, $\mathcal{H}\left(x, \mathbf{b}_{\perp}\right)$ is known to be positive. Therefore, adding the $b^{x}$-derivative of $\mathcal{E}\left(x, \mathbf{b}_{\perp}\right)$ to $\mathcal{H}\left(x, \mathbf{b}_{\perp}\right)$ has the effect of shifting the distribution towards negative $b^{x}$. For negative $\kappa_{q}$ the effect is reversed. This is why the sign of $\kappa_{q}$ determines the sign of the distortion of the quark distribution in impact parameter space. For a nucleon that is polarized in the $+\hat{y}$ direction the distortion is towards negative $\hat{x}$ when $\kappa_{q}>0$ and towards positive $\hat{x}$ when $\kappa_{q}<0$.

Note that although the argument hinges somewhat on assumptions about the shape of $E_{q}\left(x, 0, \Delta_{\perp}^{2}\right)$, these assumptions seem to be satisfied for typical model ansätze for GPDs and therefore the result is actually rather general. In the specific example of the scalar diquark model, we can calculate $\mathcal{E}_{q}\left(x, \mathbf{b}_{\perp}\right)$ and verify that the model satisfied the above assumptions.

Since the scalar diquark model provides us also with the light-cone wavefunction for the 'nucleon' there is actually a more direct way to determine the sign of the distortion in impact parameter space. For this purpose we consider the wavefunction of a quark that is polarized in the $+\hat{y}$-direction $[15,16]$

$$
\begin{aligned}
\tilde{\psi}_{+\frac{1}{2}}^{+\hat{y}}\left(x, \mathbf{k}_{\perp}\right) & \equiv \frac{1}{\sqrt{2}}\left[\tilde{\psi}_{+\frac{1}{2}}^{\uparrow}\left(x, \mathbf{k}_{\perp}\right)+i \tilde{\psi}_{+\frac{1}{2}}^{\downarrow}\left(x, \mathbf{k}_{\perp}\right)\right] \\
& =\frac{1}{\sqrt{2}}\left[M+\frac{m}{x}+\frac{i k^{x}+k^{y}}{x}\right] \tilde{\phi}\left(x, \mathbf{k}_{\perp}\right) \\
\tilde{\psi}_{-\frac{1}{2}}^{+\hat{y}}\left(x, \mathbf{k}_{\perp}\right) & \equiv \frac{1}{\sqrt{2}}\left[\tilde{\psi}_{-\frac{1}{2}}^{\uparrow}\left(x, \mathbf{k}_{\perp}\right)+i \tilde{\psi}_{-\frac{1}{2}}^{\downarrow}\left(x, \mathbf{k}_{\perp}\right)\right]
\end{aligned}
$$




$$
=\frac{1}{\sqrt{2}}\left[i\left(M+\frac{m}{x}\right)-\frac{k^{x}+i k^{y}}{x}\right] \tilde{\phi}\left(x, \mathbf{k}_{\perp}\right) .
$$

Although this should be evident from time-reversal invariance, we note that the naive (i.e. gauge noninvariant) unintegrated momentum space distribution obtained from the wavefunction [described by Eqs. $(14,15)$ ] squared is even in $\mathbf{k}_{\perp}$. Indeed

$$
\widetilde{q}_{\hat{y}}\left(x, \mathbf{k}_{\perp}\right)=\frac{1}{4 \pi}\left(\left|\tilde{\psi}_{+\frac{1}{2}}^{+\hat{y}}\left(x, \mathbf{k}_{\perp}\right)\right|^{2}+\left|\tilde{\psi}_{-\frac{1}{2}}^{+\hat{y}}\left(x, \mathbf{k}_{\perp}\right)\right|^{2}\right)=\frac{1}{4 \pi}\left[\left(M+\frac{m}{x}\right)^{2}+\frac{\mathbf{k}_{\perp}^{2}}{x^{2}}\right] \tilde{\phi}^{2},
$$

where we used the fact that $\tilde{\phi}$ is real. (See Eq. (38) in Appendix A.)

However, a transverse asymmetry in position space is not excluded by timereversal invariance and Eqs. $(14,15)$ do in fact correspond to a state with an asymmetry in the $\hat{x}$-direction as we will now demonstrate explicitly. After performing a Fourier transformation to the transverse relative position space coordinate $\mathbf{c}_{\perp}$, we have

$$
\begin{aligned}
\psi_{+\frac{1}{2}}^{+\hat{y}}\left(x, \mathbf{c}_{\perp}\right) & \equiv \int \frac{d^{2} \mathbf{k}_{\perp}}{(2 \pi)^{2}} e^{i \mathbf{c}_{\perp} \cdot \mathbf{k}_{\perp}} \tilde{\psi}_{+\frac{1}{2}}^{+\hat{y}}\left(x, \mathbf{k}_{\perp}\right) \\
& =\frac{1}{\sqrt{2}}\left[M+\frac{m}{x}+\frac{1}{x} \frac{d}{d c^{x}}-\frac{i}{x} \frac{d}{d c^{y}}\right] \phi\left(\mathbf{c}_{\perp}\right) \\
\psi_{-\frac{1}{2}}^{+\hat{y}}\left(x, \mathbf{c}_{\perp}\right) & \equiv \int \frac{d^{2} \mathbf{k}_{\perp}}{(2 \pi)^{2}} e^{i \mathbf{c}_{\perp} \cdot \mathbf{k}_{\perp}} \tilde{\psi}_{-\frac{1}{2}}^{+\hat{y}}\left(x, \mathbf{k}_{\perp}\right) \\
& =\frac{1}{\sqrt{2}}\left[\frac{i}{x} \frac{d}{d c^{x}}-\frac{1}{x} \frac{d}{d c^{y}}+i\left(M+\frac{m}{x}\right)\right] \phi\left(\mathbf{c}_{\perp}\right)
\end{aligned}
$$

with

$$
\begin{aligned}
\phi\left(\mathbf{c}_{\perp}\right) & \equiv \int \frac{d^{2} \mathbf{k}_{\perp}}{(2 \pi)^{2}} e^{i \mathbf{c}_{\perp} \cdot \mathbf{k}_{\perp}} \tilde{\phi}\left(\mathbf{k}_{\perp}\right) \\
& =-g x \sqrt{1-x} \int \frac{d^{2} \mathbf{k}_{\perp}}{(2 \pi)^{2}} e^{i \mathbf{c}_{\perp} \cdot \mathbf{k}_{\perp}} \frac{1}{\mathbf{k}_{\perp}^{2}+B} \\
& =-\frac{g}{2 \pi} x \sqrt{1-x} K_{0}\left(\left|c_{\perp}\right| \sqrt{B}\right),
\end{aligned}
$$

and

$$
B=x(1-x)\left(-M^{2}+\frac{m^{2}}{x}+\frac{\lambda^{2}}{1-x}\right) .
$$


In the limit $\left|c_{\perp}\right| \sqrt{B} \rightarrow 0$, (19) becomes $(g / 4 \pi) x \sqrt{1-x} \ln \left(\mathbf{c}_{\perp}^{2} B\right)$. Using (17) and (18), we have

$$
q_{\hat{y}}\left(x, \mathbf{c}_{\perp}\right)=\frac{1}{4 \pi}\left(\left|\psi_{+\frac{1}{2}}^{+\hat{y}}\left(x, \mathbf{c}_{\perp}\right)\right|^{2}+\left|\psi_{-\frac{1}{2}}^{+\hat{y}}\left(x, \mathbf{c}_{\perp}\right)\right|^{2}\right)
$$

with

$$
\begin{aligned}
\left|\psi_{+\frac{1}{2}}^{+\hat{y}}\left(x, \mathbf{c}_{\perp}\right)\right|^{2}=\left|\psi_{-\frac{1}{2}}^{+\hat{y}}\left(x, \mathbf{c}_{\perp}\right)\right|^{2}= & \frac{1}{2}\left\{\left[\left(M+\frac{m}{x}\right) \phi+\frac{1}{x} \frac{d}{d c^{x}} \phi\right]^{2}+\frac{1}{x^{2}}\left(\frac{d}{d c^{x}} \phi\right)^{2}\right\} \\
= & \frac{1}{2}\left\{\left(M+\frac{m}{x}\right) \phi^{2}+\frac{1}{x^{2}}\left[\left(\frac{d}{d c^{x}} \phi\right)^{2}+\left(\frac{d}{d c^{y}} \phi\right)^{2}\right]\right\} \\
& +\frac{1}{x}\left(M+\frac{m}{x}\right) \phi \frac{d}{d c^{x}} \phi
\end{aligned}
$$

since $\phi\left(\mathbf{c}_{\perp}\right)$ is real. In (22) the last term is odd under $c^{x} \rightarrow-c^{x}$ and describes the deformation of the target in impact parameter space as predicted by Eq. (11):

$$
\frac{1}{2 M} \frac{\partial}{\partial b^{x}} \mathcal{E}\left(x, \mathbf{b}_{\perp}\right)=\frac{1}{4 \pi} \frac{1}{(1-x)^{2}} \frac{2}{x}\left(M+\frac{m}{x}\right) \phi \frac{d}{d c^{x}} \phi .
$$

Since $\phi\left(x, \mathbf{c}_{\perp}\right)$ is a monotonically decreasing function of $\mathbf{c}_{\perp}^{2}$, this implies that $\left|\psi_{ \pm \frac{1}{2}}^{+\hat{y}}\left(x, \mathbf{c}_{\perp}\right)\right|^{2}$ distorted towards negative $c^{x}$. For example, the mean $\perp$ coordinate for fixed $x$ yields

$$
\begin{aligned}
\left\langle c^{x}\right\rangle & \equiv \int d^{2} \mathbf{c}_{\perp} \frac{1}{4 \pi}\left|\psi_{ \pm \frac{1}{2}}^{+\hat{y}}\left(x, \mathbf{c}_{\perp}\right)\right|^{2} c^{x} \\
& =-\frac{1}{4 \pi} \frac{1}{2 x}\left(M+\frac{m}{x}\right) \int d^{2} \mathbf{c}_{\perp} \phi^{2}<0
\end{aligned}
$$

\section{SSA in impact parameter space (scalar diquark model)}

The SSA for the scalar diquark model has been calculated in Ref. [1]. For a target that is polarized in the $+\hat{y}$ direction, one finds for the $\perp$ momentum distribution of the outgoing quarks ${ }^{\ddagger}$ of quarks that carried light-cone momentum fraction $x$

$$
\mathcal{P}^{\hat{y}}\left(x, \mathbf{r}_{\perp}\right) f_{1}\left(x, \mathbf{r}_{\perp}\right)
$$

\footnotetext{
${ }^{\ddagger}$ Note that there should be an additional overall minus sign in front of Eq. (21) of Ref. [1].
} 


$$
=C(x M+m)(1-x) \frac{1}{\mathbf{r}_{\perp}^{2}+B} \int \frac{d^{2} \mathbf{k}_{\perp}}{(2 \pi)^{2}} \frac{1}{\mathbf{k}_{\perp}^{2}+B} \frac{\left(k_{\perp}-r_{\perp}\right)^{x}}{\left(\mathbf{k}_{\perp}-\mathbf{r}_{\perp}\right)^{2}+\lambda_{g}{ }^{2}},
$$

where $C=g^{2} e_{1} e_{2} /\left(2(2 \pi)^{3}\right)$ and $B$ is given in (20). Here $\mathcal{P}^{\hat{y}}\left(x, \mathbf{r}_{\perp}\right)$ is the actual spin asymmetry and $f_{1}\left(x, \mathbf{r}_{\perp}\right)$ is the unpolarized quark distribution so that the product $\mathcal{P}^{\hat{y}} f_{1}$ is the spin-odd part of the outgoing quark momentum distribution.

In the following we are going to investigate within the context of the scalar diquark model if this SSA can be related to GPDs and the asymmetry of PDFs in impact parameter space, as has been conjectured in Ref. [7]. Eq. (25) yields for the average transverse momentum in the $\hat{x}$ direction

$$
\begin{aligned}
\left\langle r^{x}\right\rangle & \equiv \int d^{2} \mathbf{r}_{\perp} r^{x} \mathcal{P}^{\hat{y}}\left(x, \mathbf{r}_{\perp}\right) f_{1}\left(x, \mathbf{r}_{\perp}\right) \\
& =C(x M+m)(1-x) \int d^{2} \mathbf{r}_{\perp} \int \frac{d^{2} \mathbf{k}_{\perp}}{(2 \pi)^{2}} \frac{r_{\perp}^{x}}{\mathbf{r}_{\perp}^{2}+B} \frac{1}{\mathbf{k}_{\perp}^{2}+B} \frac{\left(k_{\perp}-r_{\perp}\right)^{x}}{\left(\mathbf{k}_{\perp}-\mathbf{r}_{\perp}\right)^{2}+\lambda_{g}{ }^{2}}
\end{aligned}
$$

Using Eq. (19)

$$
\frac{1}{\mathbf{k}_{\perp}^{2}+B}=-\frac{1}{g x \sqrt{1-x}} \int d^{2} \mathbf{c}_{\perp}^{\prime} e^{i \mathbf{k}_{\perp} \cdot \mathbf{c}_{\perp}^{\prime} \phi}\left(\mathbf{c}_{\perp}^{\prime}\right)
$$

and

$$
\frac{1}{\mathbf{r}_{\perp}^{2}+B}=-\frac{1}{g x \sqrt{1-x}} \int d^{2} \mathbf{c}_{\perp} e^{-i \mathbf{r}_{\perp} \cdot \mathbf{c}_{\perp}} \phi\left(\mathbf{c}_{\perp}\right)
$$

as well as the relation (46) given in Appendix B, from (25) we get

$$
\begin{aligned}
\left\langle r^{x}\right\rangle= & \frac{e_{1} e_{2}}{2(2 \pi)^{3}} \frac{(x M+m)}{x^{2}} \int d^{2} \mathbf{r}_{\perp} \int \frac{d^{2} \mathbf{k}_{\perp}}{(2 \pi)^{2}} \int d^{2} \mathbf{c}_{\perp} e^{-i \mathbf{r}_{\perp} \cdot \mathbf{c}_{\perp}} \int d^{2} \mathbf{c}_{\perp}^{\prime} e^{i \mathbf{k}_{\perp} \cdot \mathbf{c}_{\perp}^{\prime}} \\
& \times \phi\left(\mathbf{c}_{\perp}^{\prime}\right)\left(-i \frac{\partial}{\partial c_{x}}\right) \phi\left(\mathbf{c}_{\perp}\right) \frac{\left(k_{\perp}-r_{\perp}\right)^{x}}{\left(\mathbf{k}_{\perp}-\mathbf{r}_{\perp}\right)^{2}+\lambda_{g}{ }^{2}} \\
= & \frac{-i e_{1} e_{2}}{2(2 \pi)} \frac{(x M+m)}{x^{2}} \int d^{2} \mathbf{c}_{\perp} \phi\left(\mathbf{c}_{\perp}\right) \frac{\partial}{\partial c_{x}} \phi\left(\mathbf{c}_{\perp}\right) \int \frac{d^{2} \mathbf{k}_{\perp}}{(2 \pi)^{2}} e^{i \mathbf{k}_{\perp} \cdot \mathbf{c}_{\perp}} \frac{k_{\perp}^{x}}{\mathbf{k}_{\perp}^{2}+\lambda_{g}{ }^{2}} \\
= & \int d^{2} \mathbf{b}_{\perp} \mathbf{I}_{\perp}^{x}\left(\mathbf{c}_{\perp}\right) \frac{1}{2 M} \frac{\partial}{\partial b^{x}} \mathcal{E}\left(x, \mathbf{b}_{\perp}\right),
\end{aligned}
$$

where

$$
\begin{aligned}
\mathbf{I}_{\perp}^{x}\left(\mathbf{c}_{\perp}\right) & \equiv-i \frac{e_{1} e_{2}}{2} \int \frac{d^{2} \mathbf{k}_{\perp}}{(2 \pi)^{2}} e^{i \mathbf{c}_{\perp} \cdot \mathbf{k}_{\perp}} \frac{k_{\perp}^{x}}{\mathbf{k}_{\perp}^{2}+\lambda_{g}{ }^{2}} \\
& \stackrel{\lambda_{g} \rightarrow 0}{\longrightarrow} \frac{e_{1} e_{2}}{4 \pi} \frac{c_{\perp}^{x}}{\mathbf{c}_{\perp}^{2}}
\end{aligned}
$$


is the transverse impulse as a function of the $\perp$ coordinate of the struck quark.

The physical interpretation as a 'transverse impulse' becomes clear when one compares $\mathbf{I}_{\perp}$ to the net transverse impulse $\int d t \mathbf{F}_{\perp}$ that one obtains when one integrates the transverse component of the Coulomb force along a straight line from position $\left(c_{\perp}^{x}, c_{\perp}^{y}, 0\right)$ to $\left(c_{\perp}^{x}, c_{\perp}^{y},+\infty\right)$ along a straight line (remember: for an ultrarelativistic particle, $z(t)=t$ )

$$
\int_{0}^{\infty} d t \mathbf{F}_{\perp}\left(\mathbf{c}_{\perp}, z(t)\right)=\frac{e_{1} e_{2}}{4 \pi} \int_{0}^{\infty} d t \frac{\mathbf{c}_{\perp}}{\left(\mathbf{c}_{\perp}^{2}+t^{2}\right)^{\frac{3}{2}}}=\frac{e_{1} e_{2}}{4 \pi} \frac{\mathbf{c}_{\perp}}{\mathbf{c}_{\perp}^{2}}
$$

We note that Eq. (29) can be written as

$$
\begin{aligned}
\left\langle r^{x}\right\rangle & =\int d^{2} \mathbf{b}_{\perp} \mathbf{I}_{\perp}^{x}\left(\mathbf{c}_{\perp}\right) \frac{1}{2 M} \frac{\partial}{\partial b^{x}} \mathcal{E}\left(x, \mathbf{b}_{\perp}\right) \\
& =\int d^{2} \mathbf{b}_{\perp} \mathbf{I}_{\perp}^{x}\left(\mathbf{c}_{\perp}\right) q_{\hat{y}}^{\text {asym }}\left(x, \mathbf{b}_{\perp}\right) \\
& =\int d^{2} \mathbf{b}_{\perp} \mathbf{I}_{\perp}^{x}\left(\mathbf{c}_{\perp}\right)\left[q_{\hat{y}}^{\text {sym }}\left(x, \mathbf{b}_{\perp}\right)+q_{\hat{y}}^{\text {asym }}\left(x, \mathbf{b}_{\perp}\right)\right] \\
& =\int d^{2} \mathbf{b}_{\perp} \mathbf{I}_{\perp}^{x}\left(\mathbf{c}_{\perp}\right) q_{\hat{y}}\left(x, \mathbf{b}_{\perp}\right),
\end{aligned}
$$

where $q_{\hat{y}}^{\mathrm{sym}}\left(x, \mathbf{b}_{\perp}\right)$ and $q_{\hat{y}}^{\text {asym }}\left(x, \mathbf{b}_{\perp}\right)$ are symmetric and asymmetric about $b_{\perp}^{x}=0$, and they are given by $q_{\hat{y}}^{\text {sym }}\left(x, \mathbf{b}_{\perp}\right)=\mathcal{H}\left(x, \mathbf{b}_{\perp}\right)$ and $q_{\hat{y}}^{\text {asym }}\left(x, \mathbf{b}_{\perp}\right)=\frac{1}{2 M} \frac{\partial}{\partial b^{x}} \mathcal{E}\left(x, \mathbf{b}_{\perp}\right)$. This relation between GPDs and SSAs, which we now derived explicitly for the scalar diquark model, is exactly of the form "SSA $=G P D * F S I$ " that was proposed in Ref. [7].

We emphasize that $q_{\hat{y}}^{\text {asym }}\left(x, \mathbf{b}_{\perp}\right)$ is not zero in the impact parameter space and this fact made the expressions given in Eq. (29) and (33) possible, however, $\widetilde{q}_{\hat{y}}^{\text {asym }}\left(x, \mathbf{k}_{\perp}\right)$ in the momentum space is identically zero:

$$
\widetilde{q}_{\hat{y}}\left(x, \mathbf{k}_{\perp}\right)=\widetilde{q}_{\hat{y}}^{\mathrm{sym}}\left(x, \mathbf{k}_{\perp}\right),
$$

as we demonstrated explicitly in Eq. (16) in the scalar diquark model which has the wavefunctions given in Eqs. (37) and (39). Even though there exists such a difference between the asymmetric parts of $q_{\hat{y}}\left(x, \mathbf{b}_{\perp}\right)$ and $\widetilde{q}_{\hat{y}}\left(x, \mathbf{k}_{\perp}\right)$, they satisfy the following relation which is consequence of a general property of Fourier transforms:

$$
\int d^{2} \mathbf{b}_{\perp} q_{\hat{y}}\left(x, \mathbf{b}_{\perp}\right)=\int \frac{d^{2} \mathbf{k}_{\perp}}{(2 \pi)^{2}} \widetilde{q}_{\hat{y}}\left(x, \mathbf{k}_{\perp}\right),
$$


i.e., the norm (integral of the absolute square of the function) is invariant under a Fourier transform.

Our calculations also illustrate explicitely a very important difference between impact parameter dependent parton distributions $q_{\hat{y}}\left(x, \mathbf{b}_{\perp}\right)$ and unintegrated parton distributions $\tilde{q}_{\hat{y}}\left(x, \mathbf{k}_{\perp}\right)$ for a transversely polarized target: Even for a transversely polarized target the unintegrated parton distribution $\tilde{q}_{\hat{y}}\left(x, \mathbf{k}_{\perp}\right)$ has to be symmetric under $\mathbf{k}_{\perp} \rightarrow-\mathbf{k}_{\perp}$ because of parity and time-reversal arguments. Roughly speaking, the reason is that given only the spin vector $\vec{S}$ and the momentum vector $\vec{P}$ of the target it is not possible to construct a parity and time-reversal invariant correlation between the target spin and the quark momentum $\vec{k}$ that is odd under $\vec{k} \rightarrow-\vec{k}$. For example, although the term $\vec{k} \cdot(\vec{S} \times \vec{P})$ is invariant under parity, it is odd under timereversal and therefore cannot appear in the momentum distribution for the quark ${ }^{\S}$. However, the situation is different for the transverse position $\mathbf{b}_{\perp}$ where an asymmetry with respect to $\mathbf{b}_{\perp} \rightarrow-\mathbf{b}_{\perp}$ is allowed: To see this we consider the term $\vec{b} \cdot(\vec{S} \times \vec{P})$ which is clearly invariant under both parity and time-reversal. The fact that one can write down a term proportional to the spin that is odd in $\vec{b}$ and consistent with the symmetries of QCD means that a transverse (relative to $\vec{P}$ and $\vec{S}$ ) asymmetry in the position space distribution is allowed.

The explicit model calculations in the scalar diquark model confirm these general considerations since they yield $\tilde{q}_{\hat{y}}\left(x,-\mathbf{k}_{\perp}\right)=\tilde{q}_{\hat{y}}\left(x, \mathbf{k}_{\perp}\right)$ [Eq. (16)], while $q_{\hat{y}}\left(x,-\mathbf{b}_{\perp}\right) \neq$ $q_{\hat{y}}\left(x, \mathbf{b}_{\perp}\right)[$ Eq. $(24)]$.

We should also point out that Eq. (33) is consistent with expressions that have been written down previously for the mean transverse momentum arising from the final state interactions $[17,18]$. What is new is that we are evaluating these expressions in impact parameter space and for a specific model for the final state interactions.

\footnotetext{
$\S$ This argument is also the reason why a (time-reversal invariance breaking) initial or final state interaction is crucial for the Sivers asymmetry in QCD.
} 


\section{Summary}

We have analyzed the single-spin asymmetry in semi-inclusive deep inelastic scattering (DIS) in the scalar diquark model. In the impact parameter representation, the SSA emerges as a correlation between the distribution of partons in the transverse plane and the transverse impulse, which the quark being ejected from a certain transverse position has acquired as a result of the final state interactions.

The scalar diquark model is a model where both the light-cone wavefunctions of the quarks as well as the final state interactions are constructed perturbatively and therefore the constructed amplitudes still have all Lorentz symmetries as well as gauge invariance built in. Because of all these features, the physics of the Wilson line phase factor that describes the final state interaction in semi-inclusive DIS should be correctly represented in this model. In this model we obtained in the impact parameter space the formulas (29) and (33) which show explicitly the connection between the SSA and $\mathcal{E}\left(x, \mathbf{b}_{\perp}\right)$ which is the Fourier transform of the generalized parton distribution $E_{q}\left(x, 0,-\boldsymbol{\Delta}_{\perp}^{2}\right)$. These formulas are also useful for understanding the role of the orbital angular momentum in the Sivers asymmetry, since $E_{q}\left(x, 0,-\Delta_{\perp}^{2}\right)$ is given by the overlap integrals between light-cone wavefunctions whose orbital angular momenta differ by $\Delta L^{z}= \pm 1[1,14,16]$.

Without the final state interaction, one should not find a transverse momentum asymmetry because of time-reversal invariance. However, time-reversal invariance does not exclude a transverse position space asymmetry when the nucleon is polarized transversely. Our explicit calculations within the context of the scalar diquark model confirm these general predictions. We also find that the transverse position space asymmetry is described by the Fourier transform of the generalized parton

distribution $E\left(x, 0,-\Delta_{\perp}^{2}\right)$ as one would expect [12]. The transverse single-spin asymmetry in the model is obtained by convoluting the final state interaction kernel with the momentum space light-cone wavefunctions of the 'quarks' in the 'nucleon'. Upon Fourier transforming the SSA to impact parameter space, one finds that the average 
SSA has a probabilistic interpretation in impact parameter space in the sense that the asymmetry can be obtained by averaging the transverse impulse for each point in impact parameter space with the probability density to find a quark at that impact parameter. On the one hand, this result illustrates clearly the physical mechanism for the results in Ref. [1], and on the other hand it provides a specific example that confirms the general mechanism for SSA, that was proposed in Refs. [6, 7].

\section{A Scalar diquark model}

One of the virtues of light-cone wavefunctions is that many form factors and transition matrix elements have very simple representations as overlap integrals involving these wavefunctions $[19,20]$. Of course, in order to apply these overlap integrals, one needs to know the light-cone wavefunctions for each Fock component. Since we do not know the light-cone wavefunctions for the nucleon in QCD, but would still like to use them to illustrate the connection between transverse single-spin asymmetries and impact parameter space distributions, we take them from a simple toy model (a scalar diquark model).

In the scalar diquark model, the light-cone wavefunction for the two-particle Fock component is obtained by calculating the 'splitting' of a fermion into another fermion plus a scalar perturbatively. This yields the two-particle Fock component wavefunctions of the $J^{z}=+\frac{1}{2}$ state $[15,16]$ :

$$
\begin{aligned}
& \left|\Psi_{\text {two particle }}^{\uparrow}\left(P^{+}, \mathbf{P}_{\perp}=\mathbf{0}_{\perp}\right)\right\rangle \\
= & \int \frac{d^{2} \mathbf{k}_{\perp} d x}{\sqrt{x(1-x)} 16 \pi^{3}}\left[\widetilde{\psi}_{+\frac{1}{2}}^{\uparrow}\left(x, \mathbf{k}_{\perp}\right)\left|+\frac{1}{2} ; x P^{+}, \mathbf{k}_{\perp}\right\rangle+\widetilde{\psi}_{-\frac{1}{2}}^{\uparrow}\left(x, \mathbf{k}_{\perp}\right)\left|-\frac{1}{2} ; x P^{+}, \mathbf{k}_{\perp}\right\rangle\right]
\end{aligned}
$$

where

$$
\left\{\begin{array}{l}
\widetilde{\psi}_{+\frac{1}{2}}^{\uparrow}\left(x, \mathbf{k}_{\perp}\right)=\left(M+\frac{m}{x}\right) \widetilde{\phi} \\
\widetilde{\psi}_{-\frac{1}{2}}^{\uparrow}\left(x, \mathbf{k}_{\perp}\right)=-\frac{\left(k^{x}+i k^{y}\right)}{x} \widetilde{\phi}
\end{array}\right.
$$


and

$$
\widetilde{\phi}=\widetilde{\phi}\left(x, \mathbf{k}_{\perp}\right)=\frac{g}{\sqrt{1-x}} \frac{1}{M^{2}-\frac{\mathbf{k}_{\perp}^{2}+m^{2}}{x}-\frac{\mathbf{k}_{\perp}^{2}+\lambda^{2}}{1-x}} .
$$

In general one normalizes the Fock state to unit probability.

Similarly, the $J^{z}=-\frac{1}{2}$ two-particle Fock state has components:

$$
\left\{\begin{array}{l}
\widetilde{\psi}_{+\frac{1}{2}}^{\downarrow}\left(x, \mathbf{k}_{\perp}\right)=\frac{\left(k^{x}-i k^{y}\right)}{x} \widetilde{\phi} \\
\widetilde{\psi}_{-\frac{1}{2}}^{\downarrow}\left(x, \mathbf{k}_{\perp}\right)=\left(M+\frac{m}{x}\right) \widetilde{\phi}
\end{array}\right.
$$

The spin-flip amplitudes in (37) and (39) have orbital angular momentum projection $l^{z}=+1$ and -1 respectively. The $\mathbf{k}_{\perp}$ dependence in the numerators of the wavefunctions is characteristic of the orbital angular momentum, and holds for both perturbative and non-perturbative couplings.

\section{B Useful relations for Fourier transformations}

The fact that certain amplitudes that are convolutions in momentum space become diagonal in position space can be easily understood on the basis of some elementary theorems about convolutions and Fourier transforms. For example, if

$$
\begin{aligned}
f\left(\mathbf{k}_{\perp}\right) & =\int d^{2} \mathbf{b}_{\perp} e^{-i \mathbf{k}_{\perp} \cdot \mathbf{b}_{\perp}} \tilde{f}\left(\mathbf{b}_{\perp}\right) \\
g\left(\mathbf{k}_{\perp}\right) & =\int d^{2} \mathbf{b}_{\perp} e^{-i \mathbf{k}_{\perp} \cdot \mathbf{b}_{\perp}} \tilde{g}\left(\mathbf{b}_{\perp}\right)
\end{aligned}
$$

then the "form factor"

$$
F\left(\mathbf{q}_{\perp}\right) \equiv \int \frac{d^{2} \mathbf{k}_{\perp}}{(2 \pi)^{2}} f^{*}\left(\mathbf{k}_{\perp}\right) g\left(\mathbf{k}_{\perp}+\mathbf{q}_{\perp}\right)
$$

becomes diagonal in Fourier space

$$
\int \frac{d^{2} \mathbf{q}_{\perp}}{(2 \pi)^{2}} e^{i \mathbf{q}_{\perp} \cdot \mathbf{b}_{\perp}} F\left(\mathbf{q}_{\perp}\right)=\tilde{f}^{*}\left(\mathbf{b}_{\perp}\right) \tilde{g}\left(\mathbf{b}_{\perp}\right) .
$$

This well-known result forms the basis for the interpretation of non-relativistic form factors as charge distributions in position space. 
Similarly, the convolution describing the FSI in the amplitude has the form

$$
A\left(\mathbf{k}_{\perp}\right)=\int \frac{d^{2} \mathbf{q}_{\perp}}{(2 \pi)^{2}} K\left(\mathbf{k}_{\perp}-\mathbf{q}_{\perp}\right) g\left(\mathbf{q}_{\perp}\right)
$$

and thus becomes diagonal in position space

$$
\tilde{A}\left(\mathbf{b}_{\perp}\right) \equiv \int \frac{d^{2} \mathbf{k}_{\perp}}{(2 \pi)^{2}} e^{-i \mathbf{k}_{\perp} \cdot \mathbf{b}_{\perp}} A\left(\mathbf{k}_{\perp}\right)=\tilde{K}\left(\mathbf{b}_{\perp}\right) \tilde{g}\left(\mathbf{b}_{\perp}\right),
$$

where

$$
\tilde{K}\left(\mathbf{b}_{\perp}\right) \equiv \int \frac{d^{2} \mathbf{q}_{\perp}}{(2 \pi)^{2}} K\left(\mathbf{q}_{\perp}\right) e^{-i \mathbf{q}_{\perp} \cdot \mathbf{b}_{\perp}}
$$

is the Fourier transform of the kernel.

Finally, the mean SSA in momentum space contains a double convolution:

$$
\begin{aligned}
\left\langle\mathbf{k}_{\perp}\right\rangle & =\int \frac{d^{2} \mathbf{k}_{\perp}}{(2 \pi)^{2}} \int \frac{d^{2} \mathbf{q}_{\perp}}{(2 \pi)^{2}} f^{*}\left(\mathbf{q}_{\perp}\right) K\left(\mathbf{k}_{\perp}-\mathbf{q}_{\perp}\right) g\left(\mathbf{q}_{\perp}\right) \\
& =\int d^{2} \mathbf{b}_{\perp} \tilde{f}^{*}\left(\mathbf{b}_{\perp}\right) \tilde{K}\left(\mathbf{b}_{\perp}\right) \tilde{g}\left(\mathbf{b}_{\perp}\right) .
\end{aligned}
$$

Of course, for practical purposes it is often more convenient to stay in momentum space since experiments measure momenta and Fourier transforms are numerically awkward. However, for the purpose of physical interpretation, the position space expressions are useful since they are diagonal and therefore allow a probabilistic interpretation.

\section{Acknowledgements}

We thank Daniel Boer, Stan Brodsky, and Markus Diehl for very helpful comments. M.B. was supported by the DOE under grant number DE-FG03-95ER40965.

\section{References}

[1] S.J. Brodksy, D.S. Hwang and I. Schmidt, Phys. Lett. B 530, 99 (2002). 
[2] D.W. Sivers, Phys. Rev. D 43, 261 (1991).

[3] J.C. Collins, Phys. Lett. B 536, 43 (2002).

[4] X. Ji and F. Yuan, Phys. Lett. B 543, 66 (2002).

[5] A. Belitsky, X. Ji and F. Yuan, Nucl. Phys. B 656, 165 (2003).

[6] M. Burkardt, Phys. Rev. D 66, 114005 (2002).

[7] M. Burkardt, hep-ph/0302144.

[8] S.J. Brodksy, D.S. Hwang and I. Schmidt, Phys. Lett. B 553, 223 (2003).

[9] M. Burkardt, Phys. Rev. D 62, 071503 (2000), Erratum-ibid. D 66, 119903 (2002).

[10] D.E. Soper, Phys. Rev. D 15, 1141 (1977).

[11] M. Diehl, Eur. Phys. J. C 25, 223 (2002).

[12] M. Burkardt, Int. J. Mod. Phys. A 18, 173 (2003).

[13] M. Diehl, T. Feldmann, R. Jakob and P. Kroll, Nucl. Phys. B 596, 33 (2001), Erratum-ibid. B 605, 647 (2001).

[14] S.J. Brodksy, M. Diehl and D.S. Hwang, Nucl. Phys. B 596, 99 (2001).

[15] S.J. Brodsky and S.D. Drell, Phys. Rev. D 22, 2236 (1980).

[16] S.J. Brodsky, D.S. Hwang, B.Q. Ma and I. Schmidt, Nucl. Phys. B 593, 311 (2001).

[17] J. Qiu and G. Sterman, Phys. Rev. D 59, 014004 (1999).

[18] D. Boer, P. Mulders and F. Pijlman, hep-ph/0303034.

[19] G.P. Lepage and S.J. Brodksy, Phys. Rev. D 22, 2157 (1980).

[20] S.J. Brodsky, H.C. Pauli and S.S. Pinsky, Phys. Rept. 301, 299 (1998). 